\title{
Continuity of solutions for parametric generalized quasi-variational relation problems
}

Nguyen Van Hung

\section{Correspondence:}

ngvhungdhdt@yahoo.com

Department of Mathematics, Dong

Thap University, 783 Pham Huu

Lau Street, Ward 6, Cao Lanh City,

Vietnam

\begin{abstract}
In this article, we establish sufficient conditions for the solution sets of parametric generalized quasi-variational relation problems with the stability properties such as the upper semicontinuity, lower semi-continuity, the Hausdorff lower semicontinuity, continuity, Hausdorff continuity, and closedness. Our results improve recent existing ones in the literature.

Mathematics Subject Classification 2010: 90C31; 49J53; 49J40; $49 J 45$.

Keywords: quasi-variational relation problems, upper semicontinuity, lower semicontinuity, Hausdorff lower semicontinuity, upper semicontinuity, continuity, H-continuity, closedness
\end{abstract}

\section{Introduction and preliminaries}

Let $X, Y$ be Hausdorff topological vector spaces and $\Lambda, \Gamma, M$ be topological spaces. Let $A \subseteq X$ and $B \subseteq Y$ be nonempty sets. Let $K_{1}: A \times \Lambda \rightarrow 2^{A}, K_{2}: A \times \Lambda \rightarrow 2^{A}, T: A \times$ $A \times \Gamma \rightarrow 2^{B}$ be multifunctions and $R(x, t, y, \mu)$ be a relation linking $x \in A, t \in B, y \in$ $A$ and $\mu \in M$.

For the sake of simplicity, we adopt the following notations (see [1,2]). Letters $\mathrm{w}, \mathrm{m}$, and $\mathrm{s}$ are used for weak, middle, and strong, respectively, kinds of considered problems. For subsets $U$ and $V$ under consideration we adopt the notations

$$
\begin{array}{rll}
(u, v) \mathrm{w} U \times V & \text { means } & \forall u \in U, \exists v \in V, \\
(u, v) \mathrm{m} U \times V & \text { means } & \exists v \in V, \forall u \in U, \\
(u, v) \mathrm{s} U \times V & \text { means } \quad \forall u \in U, \forall v \in V, \\
\rho_{1}(U, V) & \text { means } U \subseteq V, \\
\rho_{2}(U, V) & \text { means } & U \cap V \neq \emptyset, \\
(u, v) \bar{w} U \times V & \text { means } & \exists u \in U, \forall v \in V \text { and similarly for } \bar{m}, \bar{s}, \\
\bar{\rho}_{1}(U, V) & \text { means } & U \nsubseteq V \text { and similarly for } \bar{\rho}_{2} .
\end{array}
$$

Let $\alpha \in\{\mathrm{w}, \mathrm{m}, \mathrm{s}\}, \bar{\alpha} \in\{\bar{w}, \bar{m}, \bar{s}\}, \rho \in\left\{\rho_{1}, \rho_{2}\right\}$, and $\bar{\rho} \in\left\{\bar{\rho}_{1}, \bar{\rho}_{2}\right\}$. We consider the following for parametric generalized quasi-variational relation problem (in short, $\left(\mathrm{QVR}_{\alpha}\right)$ ):

$\left(\mathbf{Q V R}_{\alpha}\right)$ : Find $\bar{x} \in K_{1}(\bar{x}, \lambda)$ such that $(y, t) \alpha K_{2}(\bar{x}, \lambda) \times T(\bar{x}, y, \gamma)$ satisfying

$$
R(\bar{x}, t, y, \mu) \text { holds. }
$$

For each $\lambda \in \Lambda, \gamma \in \Gamma, \mu \in M$, we let $E(\lambda):=\left\{x \in A \mid x \in K_{1}(x, \lambda)\right\}$ and let $S_{\alpha}: \Lambda \times \Gamma \times$ $M \rightarrow 2^{A}$ be a set-valued mapping such that $S_{\alpha}(\lambda, \gamma, \mu)$ is the solution set of $\left(\mathrm{QVR}_{\alpha}\right)$.

(c) 2012 Van Hung; licensee Springer. This is an Open Access article distributed under the terms of the Creative Commons Attribution License (http://creativecommons.org/licenses/by/2.0), which permits unrestricted use, distribution, and reproduction in any medium, provided the original work is properly cited. 
Throughout the article, we assume that $S_{\alpha}(\lambda, \gamma, \mu) \neq \varnothing$ for each $(\lambda, \gamma, \mu)$ in the neighborhoods $\left(\lambda_{0}, \gamma_{0}, \mu_{0}\right) \in \Lambda \times \Gamma \times M$.

The parametric generalized quasi-variational relation problems are more general than many following problems.

(a) The parametric variational relation problem (VR):

Let $A, B, X, Y, M=\Gamma=\Lambda, K_{1}, K_{2}, T, \alpha=s$ as in $\left(\mathrm{QVR}_{\alpha}\right)$. Then, $\left(\mathrm{QVR}_{\alpha}\right)$ becomes (VR) is studied in [3]:

Find $\bar{x} \in K_{1}(\bar{x}, \lambda)$ such that

$$
R(\bar{x}, t, y, \lambda) \quad \text { holds, } \forall t \in T(\bar{x}, y, \lambda), \forall y \in K_{2}(\bar{x}, \lambda) .
$$

(b) The parametric generalized quasi-variational inclusion problem $\left(\mathrm{QGVIP}_{\alpha}\right)$ :

Let $A, B, X, Y, M, \Gamma, \Lambda, K_{1}, K_{2}, T$ as in $\left(\mathrm{QVR}_{\alpha}\right)$ and let $Z$ be a Hausdorff topological vector space. Given a mapping $F: A \times B \times A \times M \rightarrow 2^{Z}$, the relation $R$ is defined as follows

$$
R(x, t, y, \mu) \text { holds iff } 0 \in F(x, t, y, \mu) .
$$

Then, $\left(\mathrm{QVR}_{\alpha}\right)$ becomes $\left(\mathrm{QGVIP}_{\alpha}\right)$

Find $\bar{x} \in K_{1}(\bar{x}, \lambda)$ such that $(y, t) \alpha K_{2}(\bar{x}, \lambda) \times T(\bar{x}, y, \gamma)$ satisfying

$$
0 \in F(\bar{x}, t, y, \mu) \text {. }
$$

(c) The parametric quasi-variational inclusion problem $\left(\mathrm{P}_{\alpha \rho}\right)$ :

Let $A, B, X, Y, M, \Gamma, \Lambda, K_{1}, K_{2}, T, R$ as in $\left(\mathrm{QVR}_{\alpha}\right)$ and let $Z$ be a Hausdorff topological vector space. Let $F: A \times B \times A \times M \rightarrow 2^{Z}$ and $G: A \times B \times A \times M \rightarrow 2^{Z}$ be multivalued mappings. The relation $R$ is defined as follows

$$
R(x, t, y, \mu) \text { holds iff } \rho(F(x, t, y, \mu), G(x, t, x, \mu)) .
$$

Then, $\left(\mathrm{QVR}_{\alpha}\right)$ becomes $\left(\mathrm{P}_{\alpha \rho}\right)$ is studied in [1,2]:

Find $\bar{x} \in K_{1}(\bar{x}, \lambda)$ such that $(y, t) \alpha K_{2}(\bar{x}, \lambda) \times T(\bar{x}, y, \gamma)$ satisfying

$$
\rho(F(\bar{x}, t, y, \mu), G(\bar{x}, t, \bar{x}, \mu)) .
$$

(d) The parametric vector quasi-equilibrium problems:

Let $A, X, M, \Gamma, \Lambda, K_{1} \equiv K_{2} \equiv K, T$ as in $\left(\mathrm{QVR}_{\alpha}\right)$ and let $Y$ be a Hausdorff topological vector space. Given a mapping $F: A \times A \times M \rightarrow 2^{Y}$ and $C \subseteq Y$ be a closed subset with nonempty interior, the relation $R$ is defined as follows

$$
R(x, t, y, \mu) \text { holds iff } \rho(F(t, y, \mu),(Y \backslash-\operatorname{intC})) .
$$

Then, $\left(\mathrm{QVR}_{\alpha}\right)$ becomes the parametric vector quasi-equilibrium problems is studied in [4]. Find $\bar{x} \in \operatorname{clK}(\bar{x}, \lambda)$ such that $(y, t) \alpha K(\bar{x}, \lambda) \times T(\bar{x}, y, \gamma)$ satisfying

$$
\rho(F(t, y, \mu),(Y \backslash-\text { intC })) \text {. }
$$

(e) The parametric multivalued vector quasi-equilibrium problems:

Let $A=B, X=Y, M=\Gamma, \Lambda, K_{1}=\mathrm{clK}, \mathrm{K}_{2}=\mathrm{K}, \mathrm{T}=\{\mathrm{t}\}$ as in $\left(\mathrm{QVR}_{\alpha}\right)$ and let $Z$ be a Hausdorff topological vector space. Given a mapping $F: A \times A \times M \rightarrow 2^{Z}$ and $C \subseteq Z$ be a closed subset with nonempty interior, the relation $R$ is defined as follows

$$
R(x, t, y, \mu) \text { holds iff } \rho(F(x, y, \mu),(Z \backslash-\operatorname{intC})) .
$$


Then, $\left(\mathrm{QVR} \mathrm{R}_{\alpha}\right)$ becomes the parametric multivalued vector quasi-equilibrium problems is studied in [5]. Find $\bar{x} \in \operatorname{clK}(\overline{\mathrm{x}}, \lambda)$ such that

$$
\rho(F(\bar{x}, y, \mu),(Z \backslash-\operatorname{intC})), \forall \mathrm{y} \in \mathrm{K}(\overline{\mathrm{x}}, \lambda) .
$$

(f) The parametric generalized vector quasi-equilibrium problems $\left(\mathrm{QEP}_{\alpha \rho}\right)$ :

Let $A, B, X, Y, M, \Gamma, \Lambda, K_{1}, K_{2}, T$ as in $\left(\mathrm{QVR}_{\alpha}\right)$ and let $Z$ be a Hausdorff topological vector space. Given a mapping $F: A \times B \times A \times M \rightarrow 2^{Z}$ and $C \subseteq Z$ be a closed subset with nonempty interior, the relation $R$ is defined as follows

$$
R(x, t, y, \mu) \text { holds iff } \rho(F(x, t, y, \mu), C) .
$$

Then, $\left(\mathrm{QVR}_{\alpha}\right)$ becomes $\left(\mathrm{QEP}_{\alpha \rho}\right)$

Find $\bar{x} \in K_{1}(\bar{x}, \lambda)$ such that $(y, t) \alpha K_{2}(\bar{x}, \lambda) \times T(\bar{x}, y, \gamma)$ satisfying

$$
\rho(F(\bar{x}, t, y, \mu), C) .
$$

Stability properties of solution sets for parametric generalized quasi-variational relation problem is an important topic in optimization theory and applications. Recently, the continuity, especially the upper semicontinuity, the lower semicontinuity and the Hausdorff lower semicontinuity of the solution sets have been investigated in models as equilibrium problems [1,2,4-13], variational inequality problems [14-19], and the references therein.

The structure of this article is as follows. In the remaining part of this section, we recall definitions for later uses. Section "Main results" is devoted to the upper semicontinuity, the lower semicontinuity, and the Hausdorff lower semicontinuity of solutions for problem $\left(\mathrm{QVR}_{\alpha}\right)$. Applications to the parametric vector quasi-equilibrium problem are presented in Section "Applications".

Now we recall some notions see [5,6,20,21]. Let $X$ and $Y$ be as above and $G: X \rightarrow 2^{Y}$ be a multifunction. $G$ is said to be lower semicontinuous (lsc) at $x_{0}$ if $G\left(x_{0}\right) \cap U \neq \varnothing$ for some open set $U \subseteq Y$ implies the existence of a neighborhood $N$ of $x_{0}$ such that, for all $x \in N, G(x) \cap U \neq \varnothing$. An equivalent formulation is that: $G$ is lsc at $x_{0}$ if $\forall x_{\alpha} \rightarrow x_{0}, \forall z_{0} \in G\left(x_{0}\right), \exists z_{\alpha} \in G\left(x_{\alpha}\right), z_{\alpha} \rightarrow z_{0} . G$ is called upper semicontinuous (usc) at $x_{0}$ if for each open set $U \supseteq G\left(x_{0}\right)$, there is a neighborhood $N$ of $x_{0}$ such that $U \supseteq G(x)$, for all $x \in N$. G is said to be Hausdorff upper semicontinuous (H-usc in short; Hausdorff lower semicontinuous, $\mathrm{H}$-lsc, respectively) at $x_{0}$ if for each neighborhood $B$ of the origin in $Y$, there exists a neighborhood $N$ of $x_{0}$ such that, $G(x) \subseteq G\left(x_{0}\right)+B, \forall x \in N$ $\left(G\left(x_{0}\right) \subseteq G(x)+B, \forall x \in N\right)$. G is said to be continuous at $x_{0}$ if it is both lsc and usc at $x_{0}$ and to be H-continuous at $x_{0}$ if it is both H-lsc and H-usc at $x_{0} . G$ is called closed at $x_{0}$ if for each net $\left\{\left(x_{\alpha}, z_{\alpha}\right)\right\}$ graph $G:=\{(x, z) \mid z \in G(x)\},\left(x_{\alpha}, z_{\alpha}\right) \rightarrow\left(x_{0}, z_{0}\right), z_{0}$ must belong to $G\left(x_{0}\right)$. We say that $G$ satisfies a certain property in a subset $A \subseteq X$ if $G$ satisfies it at every points of $A$. If $A=X$ we omit "in $X^{\prime \prime}$ in the statement.

Let $A$ and $Y$ be as above and $G: A \rightarrow 2^{Y}$ be a multifunction.

(i) If $G$ is usc at $x_{0}$, then $G$ is $H$-usc at $x_{0}$. Conversely if $G$ is $H$-usc at $x_{0}$ and if $G\left(x_{0}\right)$ is compact, then $G$ is usc at $x_{0}$;

(ii) If $G$ is H-lsc at $x_{0}$, then $G$ is lsc at $x_{0}$. The converse is true if $G\left(x_{0}\right)$ is compact;

(iii) If $Y$ is compact and $G$ is closed at $x_{0}$, then $G$ is usc at $x_{0}$;

(iv) If $G$ is usc at $x_{0}$ and $G\left(x_{0}\right)$ is closed, then $G$ is closed at $x_{0}$; 
(v) If $G$ has compact values, then $G$ is usc at $x_{0}$ if and only if, for each net $\left\{x_{\alpha}\right\} \subseteq A$ which converges to $x_{0}$ and for each net $\left\{y_{\alpha}\right\} \subseteq G\left(x_{\alpha}\right)$, there are $y_{0} \in G\left(x_{0}\right)$ and a subnet $\left\{y_{\beta}\right\}$ of $\left\{y_{\alpha}\right\}$ such that $y_{\beta} \rightarrow y_{0}$.

Now we let $A, B, X, Y, M, \Gamma, \Lambda, R$ as in $\left(\mathrm{QVR}_{\alpha}\right)$, we use the following notations for level sets of $R$

$$
\begin{aligned}
& 1 \mathrm{ev}_{\text {upper }} R:=\{(x, t, y, \mu) \mid R(x, t, y, \mu) \text { holds }\} . \\
& 1 \operatorname{ev}_{\text {upper }} R\left(., \ldots, \mu_{0}\right):=\left\{(x, t, y) \mid R\left(x, t, y, \mu_{0}\right) \text { holds }\right\} . \\
& 1 \operatorname{ev}_{\text {lower }} R:=\{(x, t, y, \mu) \mid R(x, t, y, \mu) \text { does not hold }\} .
\end{aligned}
$$

\section{Main results}

In this section, we discuss the upper semicontinuity, the lower semicontinuity, the Hausdorff lower semicontinuity, continuity, and $\mathrm{H}$-continuity of solution sets for parametric quasi-variational relation problem $\left(\mathrm{QVR}_{\alpha}\right)$.

Theorem 1 Assume for problem $\left(\mathrm{QVR}_{\alpha}\right)$ that

(i) $E$ is usc at $\lambda_{0}$ and $E\left(\lambda_{0}\right)$ is compact, and $K_{2}$ is lsc in $K_{1}(A, \Lambda) \times\left\{\lambda_{0}\right\}$;

(ii) in $K_{1}(A, \Lambda) \times K_{2}\left(K_{1}(A, \Lambda), \Lambda\right) \times\left\{\gamma_{0}\right\}, T$ is usc and compact-valued if $\alpha=w$ (or $\alpha=m)$, and lsc if $\alpha=s$;

(iii) in $K_{1}(A, \Lambda) \times T\left(K_{1}(A, \Lambda), K_{2}\left(K_{1}(A, \Lambda), \Lambda\right), \Gamma\right) \times K_{2}\left(K_{1}(A, \Lambda), \Lambda\right) \times\left\{\mu_{0}\right\}$, lev upper $R$ is closed.

Then $S_{\alpha}$ is both usc and closed at $\left(\lambda_{0}, \gamma_{0}, \mu_{0}\right)$.

Proof. Since $\alpha=\{w, m, s\}$, we have in fact three cases. However, the proof techniques are similar. We consider only the cases $\alpha=w$. We first prove that $S_{w}$ is upper semicontinuous at $\left(\lambda_{0}, \gamma_{0}, \mu_{0}\right)$. Indeed, we suppose to the contrary that $S_{w}$ is not upper semicontinuous at $\left(\lambda_{0}, \gamma_{0}, \mu_{0}\right)$, i.e., there is an open subset $U$ of $S_{w}\left(\lambda_{0}, \gamma_{0}, \mu_{0}\right)$ such that for all nets $\left\{\left(\lambda_{n}, \gamma_{n}, \mu_{n}\right)\right\}$ convergent to $\left(\lambda_{0}, \gamma_{0}, \mu_{0}\right)$, there exists $x_{n} \in S_{w}\left(\lambda_{n}, \gamma_{n}, \mu_{n}\right), x_{n} \notin$ $U, \forall n$. By the upper semicontinuity of $E$ and the compactness of $E\left(\lambda_{0}\right)$, one can assume that $x_{n} \rightarrow x_{0}$ for some $x_{0} \in E\left(\lambda_{0}\right)$. If $x_{0} \notin S_{w}\left(\lambda_{0}, \gamma_{0}, \mu_{0}\right)$, then $\exists y_{0} \in K_{2}\left(x_{0}, \lambda_{0}\right), \forall t_{0} \in T$ $\left(x_{0}, y_{0}, \gamma_{0}\right)$ such that

$$
R\left(x_{0}, t_{0}, y_{0}, \mu_{0}\right) \text { does not hold. }
$$

By the lower semicontinuity of $K_{2}$ at $\left(x_{0}, \lambda_{0}\right)$, there exists $y_{n} \in K_{2}\left(x_{n}, \lambda_{n}\right)$ such that $y_{n}$ $\rightarrow y_{0}$. Since $x_{n} \in S_{w}\left(\lambda_{n}, \gamma_{n}, \mu_{n}\right), \exists t_{n} \in T\left(x_{n}, y_{n}, \gamma_{n}\right)$ such that

$$
R\left(x_{n}, t_{n}, y_{n}, \mu_{n}\right) \text { holds. }
$$

Since $T$ is usc at $\left(x_{0}, y_{0}, \gamma_{0}\right)$ and $T\left(x_{0}, y_{0}, \gamma_{0}\right)$ is compact, there exists $t_{0} \in T\left(x_{0}, y_{0}\right.$, $\gamma_{0}$ ) such that $t_{n} \rightarrow t_{0}$ (can take a subnet if necessary). By the condition (iii) and (2), we have

$$
R\left(x_{0}, t_{0}, y_{0}, \mu_{0}\right) \text { holds, }
$$

we see a contradiction between (1) and (3). Thus, $x_{0} \in S_{w}\left(\lambda_{0}, \gamma_{0}, \mu_{0}\right) \subseteq U$, this contradicts to the fact $x_{n} \notin U, \forall n$. Hence, $S_{w}$ is upper semicontinuous at $\left(\lambda_{0}, \gamma_{0}, \mu_{0}\right)$.

Now we prove that $S_{w}$ is closed at $\left(\lambda_{0}, \gamma_{0}, \mu_{0}\right)$. Indeed, we supposed that $S_{w}$ is not closed at $\left(\lambda_{0}, \gamma_{0}, \mu_{0}\right)$, i.e., there is a net $\left\{\left(x_{n}, \lambda_{n}, \gamma_{n}, \mu_{n}\right)\right\} \rightarrow\left(x_{0}, \lambda_{0}, \gamma_{0}, \mu_{0}\right)$ with $x_{n} \in S_{w}$ $\left(\lambda_{n}, \gamma_{n}, \mu_{n}\right)$ but $x_{0} \notin S_{w}\left(\lambda_{0}, \gamma_{0}, \mu_{0}\right)$. The further argument is the same as above. And so we have $S_{w}$ is closed at $\left(\lambda_{0}, \gamma_{0}, \mu_{0}\right)$. 
The following example shows that the upper semicontinuity and the compactness of $E$ are essential.

Example 2 Let $A=B=X=Y=\mathbb{R}, \Lambda=\Gamma=M=[0,1], \lambda_{0}=0, F(x, t, y, \lambda)=2^{\lambda+1}$, $K_{1}(x, \lambda)=(-\lambda-1, \lambda], K_{2}(x, \lambda)=\{-1\}$ and $T(x, y, \lambda)=\left[0, e^{2^{\lambda}+\cos \lambda}\right]$. We let relation $R$ be defined by $R(x, t, y, \lambda)$ holds iff $F(x, t, y, \lambda) \subseteq \mathbb{R}_{+}$. Then, we have $E(0)=(-1,0]$ and $E(\lambda)=(-\lambda-1, \lambda], \forall \lambda \in(0,1]$. We show that $K_{2}$ is lsc and assumptions (ii) and (iii) of Theorem 1 are fulfilled. But $S_{\alpha}$ is neither usc nor closed at $(0,0,0)$. The reason is that $E$ is not usc at 0 and $E(0)$ is not compact. In fact, $S_{\alpha}(0,0,0)=(-1,0]$ and $S_{\alpha}(\lambda, \gamma, \mu)=$ $(-\lambda-1, \lambda], \forall \lambda \in(0,1]$.

The following example shows that the lower semicontinuity of $K_{2}$ is essential.

Example 3 Let $X, Y, \Lambda, \Gamma, M, \lambda_{0}$ as in Example 2 and let $A=B=[-3,3], F(x, t, y, \lambda)$ $=x+y+\lambda, K_{1}(x, \lambda)=[0,3], T(x, y, \lambda)=\{t\}$. Let relation $R$ be defined by $R(x, t, y, \lambda)$ holds iff $F(x, t, y, \lambda) \subseteq \mathbb{R}_{+}$and

$$
K_{2}(x, \lambda)= \begin{cases}\{-3,0,3\} & \text { if } \lambda=0, \\ \{0,3\} & \text { otherwise. }\end{cases}
$$

We have $E(\lambda)=[0,3], \forall \lambda \in[0,1]$. Hence $E$ is usc at 0 and $E(0)$ is compact and the conditions (ii) and (iii) of Theorem 1 are easily seen to be fulfilled. But $S_{\alpha}$ is not upper semicontinuous at $(0,0,0)$. The reason is that $K_{2}$ is not lower semicontinuous. In fact,

$$
S_{\alpha}(\lambda, \gamma, \mu)=\left\{\begin{array}{lll}
\{3\} \quad \text { if } \quad \lambda=0, \\
{[0,3] \text { if } \quad \lambda \in(0,1]}
\end{array}\right.
$$

The following example shows that the condition (iii) of Theorem 1 is essential.

Example 4 Let $\Lambda, \Gamma, M, T, \lambda_{0}$ as in Example 3 and let $X=Y=A=B=[0,1]$. Let relation $R$ be defined by $R(x, t, y, \lambda)$ holds iff $F(x, t, y, \lambda) \subseteq \mathbb{R}_{+}, K_{1}(x, \lambda)=K_{2}(x, \lambda)=[0,1]$ and $F(x, t, y, 0)=\frac{x}{2}-\frac{y}{2}, F(x, t, y, \lambda)=\frac{y}{2}-\frac{x}{3}, \forall \lambda \in(0,1]$. We show that the assumptions (i) and (ii) of Theorem 1 are easily seen to be fulfilled and

$$
S_{\alpha}(\lambda, \gamma, \mu)= \begin{cases}\{0\} \text { if } & \lambda \in(0,1] \\ \{1\} \text { if } & \lambda=0 .\end{cases}
$$

But $S_{\alpha}$ is not usc at $(0,0,0)$. The reason is that assumption (iii) is violated. Indeed, taking $x_{n}=0, t_{n}=0, y_{n}=\frac{1}{2}, \lambda_{n}=\frac{1}{n} \rightarrow 0$ as $n \rightarrow \infty$, then $\left\{\left(x_{n}, t_{n}, y_{n}, \lambda_{n}\right)\right\} \rightarrow\left(0,0, \frac{1}{2}, 0\right)$ and $F\left(x_{n}, t_{n}, y_{n}, \lambda_{n}\right)=F\left(0,0, \frac{1}{2}, \frac{1}{n}\right)=\frac{1}{4}>0$, but $F\left(0,0, \frac{1}{2}, 0\right)=-\frac{1}{4}<0$.

The following example shows that all assumptions of Theorem 1 are fulfilled. But Theorem 3.2 in [5] cannot be applied.

Example 5 Let $A, B, X, Y, \Lambda, \Gamma, M, \lambda_{0}$ as in Example 2 and let $\mathrm{K}_{1}(x, \lambda)=\mathrm{K}_{2}(x, \lambda)=$ $[0,1], T(x, y, \gamma)=\left[0,2^{\cos ^{6} x+\sin ^{4} x+2}\right]$ and

$$
F(x, t, y, \lambda)= \begin{cases}\{0\} & \text { if } \lambda=0, \\ 3^{\sin ^{4} x+\cos ^{2} x+2} \text { otherwise. }\end{cases}
$$

Let relation $R$ be defined by $R(x, t, y, \lambda)$ holds iff $F(x, t, y, \lambda) \subseteq \mathbb{R}_{+}$. We show that the assumptions (i), (ii), and (iii) of Theorem 1 are easily seen to be fulfilled and

$$
S_{\alpha}(\lambda, \gamma, \mu)=[0,1], \forall \lambda \in[0,1]
$$

Hence, $S_{\alpha}$ is usc at $(0,0,0)$. But Theorem 3.2 in [5] cannot be applied. The reason is that $F$ is not usc at $(x, t, y, 0)$. 
The following example shows that all assumptions of Theorem 1 are fulfilled. But Theorem 3.4 in [5] cannot be applied.

Example 6 Let $A, B, X, Y, \Lambda, \Gamma, M, \lambda_{0}$, as in, Example, 5 and, let, $K_{1}(x, \lambda),=K_{2}(x, \lambda)=$ $[0,3], T(x, y, \gamma)=\{t\}$ and

$$
F(x, t, y, \lambda)= \begin{cases}\{0\} & \text { if } \lambda=0, \\ e^{\cos ^{2} \lambda+1} & \text { otherwise }\end{cases}
$$

Let relation $R$ be defined by $R(x, t, y, \lambda)$ holds iff $F(x, t, y, \lambda) \subseteq \mathbb{R}_{+}$. We show that the assumption (i), (ii) and (iii) of Theorem 1 are easily seen to be fulfilled

Hence, $S_{\alpha}$ is usc at $(0,0,0)$. But Theorem 3.4 in [5] cannot be applied. The reason is that $F$ is not usc (x, t, y, 0$)$.

Assumptions in Theorem 1, we have $K_{2}$ is lsc in $K_{1}(A, \Lambda) \times\left\{\lambda_{0}\right\}$ (which is not imposed in this Theorem 4.1 of [10]). The Example 3 shows that the lower semicontinuity of $K_{2}$ needs to be added to Theorem 4.1 of [10].

Remark 7 (i) In the special case, if $T(x, y, \gamma)=\{t\}, \Lambda=\Gamma=M, A=B, X=Y, K_{1}=$ $K_{2}=K$ and the variational relation $R$ is defined as follows $R(x, t, y, \lambda)$ holds iff $F(x, y, \lambda)$ $\Phi$-intC $(\mathrm{x}, \lambda)$ (or $F(x, y, \lambda) \cap$-intC $(\mathrm{x}, \lambda)=\varnothing$ ), where $F: A \times A \times \Lambda \rightarrow 2^{Y}$ and $C: A \times$ $\Lambda \rightarrow 2^{Y}$ be multifunctions, with $C(x, \lambda)$ being a convex cone. Then, $\left(\mathrm{QVR}_{\alpha}\right)$ becomes (PGQVEP) and (PEQVEP) in [10].

(ii) In the special case as in Remark 7 (i). Then, Theorem 1 reduces to Theorem 4.1 in [10]. However the proof of the Theorem 4.1 in a different way. Its assumptions (i)-(iv) derive (i) Theorem 1, assumptions (v) and (vi) coincide with (iii) of Theorem 1.

The following example shows a case where the assumed compactness in Theorem 4.1 of [10] is violated but the assumptions of Theorem 1 are fulfilled.

Example 8 Let $X, Y, \Lambda, \Gamma, M, T, \lambda_{0}$, as in Example 6 and we let $A=B=[0,3)$, $F(x, y, \lambda)=x-y$ and $K_{1}(x, \lambda)=K_{2}(x, \lambda)=[1,2]$. Let relation $R$ be defined by $R(x, t$, $y, \lambda)$ holds iff $F(x, t, y, \lambda) \subseteq \mathbb{R}_{+}$. We show that the assumptions of Theorem 1 are easily seen to be fulfilled and so $S_{\alpha}$ is usc and closed at $(0,0,0)$, although $A$ is not compact. In fact, $S_{\alpha}(\lambda, \gamma, \mu)=\{2\}, \forall \lambda \in[0,1]$.

Theorem 9 Assume for problem $\left(\mathrm{QVR}_{\alpha}\right)$ that

(i) $E$ is lsc at $\lambda_{0}, K_{2}$ is usc and compact-valued in $K_{1}(A, \Lambda) \times\left\{\lambda_{0}\right\}$;

(ii) in $K_{1}(A, \Lambda) \times K_{2}\left(K_{1}(A, \Lambda), \Lambda\right) \times\left\{\gamma_{0}\right\}, T$ is usc and compact-valued if $\alpha=s$, and lsc if $\alpha=w$ (or $\alpha=m)$;

(iii) in $K_{1}(A, \Lambda) \times T\left(K_{1}(A, \Lambda), K_{2}\left(K_{1}(A, \Lambda), \Lambda\right), \Gamma\right) \times K_{2}\left(K_{1}(A, \Lambda), \Lambda\right) \times\left\{\mu_{0}\right\}, \operatorname{lev}_{\text {lower }} R$ is closed.

Then $S_{\alpha}$ is lower semicontinuous at $\left(\lambda_{0}, \gamma_{0}, \mu_{0}\right)$.

Proof. Since $\alpha=\{w, m, s\}$, we have in fact three cases. However, the proof techniques are similar. We consider only the cases $\alpha=s$. Suppose to the contrary that $S_{s}$ is not lsc at $\left(\lambda_{0}, \gamma_{0}, \mu_{0}\right)$, i.e., there are $x_{0} \in S_{s}\left(\lambda_{0}, \gamma_{0}, \mu_{0}\right)$ and net $\left\{\left(\lambda_{n}, \gamma_{n}, \mu_{n}\right)\right\},\left(\lambda_{n}, \gamma_{n}, \mu_{n}\right) \rightarrow\left(\lambda_{0}, \gamma_{0}\right.$, $\left.\mu_{0}\right)$ such that $\forall x_{n} \in S_{s}\left(\lambda_{n}, \gamma_{n}, \mu_{n}\right), x_{n} \rightarrow x_{0}$. Since $E$ is lsc at $\lambda_{0}$, there is $x_{n}^{\prime} \in E\left(\lambda_{n}\right)$ with $x_{n}^{\prime} \rightarrow x_{0}$. By the above contradiction assumption, there must be a subnet $\left\{x_{m}^{\prime}\right\}$ of $\left\{x_{n}^{\prime}\right\}$ such that, $\forall m, x_{m}^{\prime} \notin S_{s}\left(\lambda_{m}, \gamma_{m}, \mu_{m}\right)$, i.e., $\exists \gamma_{m} \in K_{2}\left(x_{m}^{\prime}, \lambda_{m}\right), \exists t_{m} \in T\left(x_{m}^{\prime}, \gamma_{m}, \gamma_{m}\right)$ such that

$$
R\left(x_{m}^{\prime}, t_{m}, y_{m}, \mu_{m}\right) \text { does not hold. }
$$


As $K_{2}$, is usc at $\left(x_{0}, \lambda_{0}\right)$ and $K_{2}\left(x_{0}, \lambda_{0}\right)$ is compact, one has $y_{0} \in K_{2}\left(x_{0}, \lambda_{0}\right)$ such that $y_{m} \rightarrow y_{0}$ (taking a subnet if necessary). By the upper semicontinuity of $T$ at $\left(x_{0}, y_{0}, \gamma_{0}\right)$, one has $t_{0} \in T\left(x_{0}, y_{0}, \gamma_{0}\right)$ such that $t_{m} \rightarrow t_{0}$.

Since $\left(x_{m}^{\prime}, t_{m}, \gamma_{m}, \lambda_{m}, \gamma_{m}, \mu_{m}\right) \rightarrow\left(x_{0}, t_{0}, \gamma_{0}, \lambda_{0}, \gamma_{0}, \mu_{0}\right)$ and by the condition (iii) and (4), yields that

$$
R\left(x_{0}, t_{0}, y_{0}, \mu_{0}\right) \text { does not hold, }
$$

which is impossible since $x_{0} \in S_{s}\left(\lambda_{0}, \gamma_{0}, \mu_{0}\right)$. Therefore, $S_{s}$ is lsc at $\left(\lambda_{0}, \gamma_{0}, \mu_{0}\right)$.

The following example shows that the lower semicontinuity of $E$ is essential

Example 10 Let $A, B, X, Y, \Lambda, \Gamma, M, \lambda_{0}$ as in Example 2 and let $F(x, t, y, \lambda)=2^{\lambda}, T$ $(x, y, \lambda)=\{t\}, K_{2}(x, \lambda)=[0,1]$. Let relation $R$ be defined by $R(x, t, y, \lambda)$ holds iff $F(x, t$, $y, \lambda) \subseteq(0,+\infty)$ and

$$
K_{1}(x, \lambda)=\left\{\begin{array}{l}
{[-1,1] \quad \text { if } \lambda=0} \\
{[-\lambda-1,0] \text { otherwise }}
\end{array}\right.
$$

We have $E(0)=[-1,1], E(\lambda)=[-\lambda-1,0], \forall \lambda \in(0,1]$. Hence $K_{2}$ is usc and the conditions (ii) and (iii) of Theorem 9 are easily seen to be fulfilled. But $S$ is not lower semicontinuous at $(0,0,0)$. The reason is that $E$ is not lower semicontinuous at 0 . In fact, $S_{\alpha}(0,0,0)=[-1,1]$ and $S_{\alpha}(\lambda, \gamma, \mu)=[-\lambda-1,0], \forall \lambda \in(0,1]$.

The following example shows that all assumptions of Theorem 9 are fulfilled. But Theorems 2.1 and 2.3 in [5] and Theorem 2.2 in [4] are not fulfilled.

Example 11 Let $A, B ; X, Y, T, \Lambda, \Gamma, M, \lambda_{0}$ as in Example 10, let $K_{1}(x, \lambda)=K_{2}(x, \lambda)=\left[0, \frac{1}{2}\right]$ and

$$
F(x, y, \lambda)=\left\{\begin{array}{l}
{\left[\frac{1}{2}, 1\right] \quad \text { if } \lambda=0,} \\
{\left[2,3^{\lambda+2}\right] \text { otherwise. }}
\end{array}\right.
$$

and we let relation $R$ be defined by $R(x, t, y, \mu)$ holds iff $F(x, y, \lambda) \subseteq(0,+\infty)$. We show that the assumptions (i), (ii) and (iii) of Theorem 9 are satisfied and $\forall \lambda \in[0,1], \forall \lambda \in[0,1]$. Theorems 2.1 and 2.3 in [5] and Theorem 2.2 in [4] are not fulfilled. The reason is that $F$ is neither usc nor lsc at $(x, y, 0)$.

Theorem 12 Impose the assumption of Theorem 9 and the following additional conditions:

(iv) $K_{2}\left(., \lambda_{0}\right)$ is lsc in $K_{1}(A, \Lambda)$ and $E\left(\lambda_{0}\right)$ is compact;

(v) in $K_{1}(A, \Lambda) \times K_{2}\left(K_{1}(A, \Lambda), \Lambda\right), T$ (., ., $\left.\gamma_{0}\right)$ is usc and compact-valued if $\alpha=w$ (or $\alpha=m)$, and lsc if $\alpha=s$;

(vi) in $K_{1}(A, \Lambda) \times T\left(K_{1}(A, \Lambda), K_{2}\left(K_{1}(A, \Lambda), \Lambda\right), \Gamma\right) \times K_{2}\left(K_{1}(A, \Lambda), \Lambda\right)$, lev upper $R\left(.\right.$, ., $\left.\mu_{0}\right)$ is closed;

Then $S_{\alpha}$ is Hausdorff lower semicontinuous at $\left(\lambda_{0}, \gamma_{0}, \mu_{0}\right)$.

Proof. We consider only for the cases $\alpha=s$. We first prove that $S_{s}\left(\lambda_{0}, \gamma_{0}, \mu_{0}\right)$ is closed. Indeed, we let $x_{n} \in S_{s}\left(\lambda_{0}, \gamma_{0}, \mu_{0}\right)$ such that $x_{n} \rightarrow x_{0}$. If $x_{0} \notin S_{s}\left(\lambda_{0}, \gamma_{0}, \mu_{0}\right)$, then $\exists y_{0} \in K_{2}\left(x_{0}, \lambda_{0}\right), \exists t_{0} \in T\left(x_{0}, y_{0}, \gamma_{0}\right)$ such that

$$
R\left(x_{0}, t_{0}, y_{0}, \mu_{0}\right) \text { does not hold. }
$$

By the lower semicontinuity of $K_{2}\left(., \lambda_{0}\right)$ at $x_{0}$, one has $y_{n} \in K_{2}\left(x_{n}, \lambda_{0}\right)$ such that $y_{n} \rightarrow$ $y_{0}$. By the lower semicontinuity of $T\left(., ., \gamma_{0}\right)$ at $\left(x_{0}, y_{0}\right)$, one has $t_{n} \in T\left(x_{n}, y_{n}, \gamma_{0}\right)$ such that $t_{n} \rightarrow t_{0}$. Since $x_{n} \in S_{s}\left(\lambda_{0}, \gamma_{0}, \mu_{0}\right)$, we have 


$$
R\left(x_{n}, t_{n}, y_{n}, \mu_{0}\right) \text { holds. }
$$

Since $\left(x_{n}, t_{n}, y_{n}\right) \rightarrow\left(x_{0}, t_{0}, y_{0}\right)$ and by the condition (vi) and (6) yields that

$$
R\left(x_{0}, t_{0}, y_{0}, \mu_{0}\right) \text { holds, }
$$

we see a contradiction between (5) and (7). Therefore, $S_{s}\left(\lambda_{0}, \gamma_{0}, \mu_{0}\right)$ is closed.

On the other hand, since $S_{s}\left(\lambda_{0}, \gamma_{0}, \mu_{0}\right) \subseteq E\left(\lambda_{0}\right)$ is compact by $E\left(\lambda_{0}\right)$ compact. Since $S_{s}$ is lower semicontinuous at $\left(\lambda_{0}, \gamma_{0}, \mu_{0}\right)$ and $S_{s}\left(\lambda_{0}, \gamma_{0}, \mu_{0}\right)$ compact. Hence $S_{s}$ is Hausdorff lower, semicontinuous at $\left(\lambda_{0}, \gamma_{0}, \mu_{0}\right)$. And so we complete the proof. $\square$

The following example shows that the assumed compactness in (iv) is essential

Example 13 Let $X=A=B=\mathbb{R}^{2}, Y=\mathbb{R}, \Lambda=\Gamma=M=[0,1], \lambda_{0}=0$, and $x=\left(x_{1}, x_{2}\right) \in \mathbb{R}^{2}$, $T(x, y, \lambda)=\left[0,3^{\sin ^{4} x+\sin ^{2} x+1}\right], T(x, y, \lambda)=\left[0,3^{\sin ^{4} x+\sin ^{2} x+1}\right]$ and

$$
F(x, t, y, \lambda)= \begin{cases}\left\{\frac{1}{2}\right\} & \text { if } \lambda=0 \\ \left\{\frac{1}{2}+\frac{\lambda}{2^{\lambda+1}}\right\} & \text { otherwise. }\end{cases}
$$

Let relation $R$ be defined by $R(x, t, y, \lambda)$ holds iff $F(x, t, y, \lambda) \subseteq(0,+\infty)$. We have $E(0)=$, $\left\{x \in \mathbb{R}^{2} \mid x^{2}=0\right\}$ and $\left.E(\lambda)=\left\{x \in \mathbb{R}^{2} \mid x_{2}=\lambda x_{1}^{4}\right)\right\}, \forall \lambda \in(0,1]$. We show that the assumptions, of Theorem 12 are satisfied, but the compactness of $E(0)$ is not satisfied. Hence, $S_{\alpha}$ is not, Hausdorff lower semicontinuous at $(0,0,0)$. In fact, $S_{\alpha}(0,0,0)=\left\{\left(x_{1}, x_{2}\right) \in \mathbb{R}^{2} \mid x_{2}=\right.$ $0\}$ and $\left.S_{\alpha}(\lambda, \gamma, \mu)=\left\{\left(x_{1}, x_{2}\right) \in \mathbb{R}^{2} \mid x_{2}=\lambda x_{1}^{4}\right)\right\}, \forall \lambda \in(0,1]$.

Corollary 14 Suppose that all conditions in Theorems 1 and 9 are satisfied. Then, we have $S \alpha$ is both continuous and closed at $\left(\lambda_{0}, \gamma_{0}, \mu_{0}\right)$.

Corollary 15 Suppose that all conditions in Theorems 1 and 12 are satisfied. Then, we have $S_{\alpha}$ is Hausdorff continuous and closed at $\left(\lambda_{0}, \gamma_{0}, \mu_{0}\right)$.

\section{Applications}

Since our generalized quasi-variational relation problems include many rather general problems as particular cases as mentioned in Section "Introduction". The results of Section "Main results" can derive corresponding to results of these special cases. In Section "Applications" we discuss only some corollaries for generalized vector quasiequilibrium problems as example.

In this section, we discuss the upper semicontinuity, the lower semicontinuity, the Hausdorff lower semicontinuity, continuity, H-continuity of solution sets for generalized parametric vector quasi-equilibrium problems $\left(\mathrm{QEP}_{\alpha \rho}\right)$.

For each $\lambda \in \Lambda, \gamma \in \Gamma, \mu \in M$, let $\Psi_{\alpha \rho}: \Lambda \times \Gamma \times M \rightarrow 2^{A}$ e a set-valued mapping such that $\Psi_{\alpha \rho}(\lambda, \gamma, \mu)$ is the solution set of $\left(\mathrm{QEP}_{\alpha \rho}\right)$.

Throughout the article, we assume that $\Psi_{\alpha \rho}(\lambda, \gamma, \mu) \neq \varnothing$ for each $(\lambda, \gamma, \mu)$ in the neighborhoods $\left(\lambda_{0}, \gamma_{0}, \mu_{0}\right) \in \Lambda \times \Gamma \times M$.

Corollary 16 Assume for problem $\left(\mathrm{QEP}_{\alpha \rho}\right)$ that

(i) $E$ is usc at $\lambda_{0}$ and $E\left(\lambda_{0}\right)$ is compact, and $K_{2}$ is lsc in $K_{1}(A, \Lambda) \times\left\{\lambda_{0}\right\}$;

(ii) in $K_{1}(A, \Lambda) \times K_{2}\left(K_{1}(A, \Lambda), \Lambda\right) \times\left\{\gamma_{0}\right\}$, T is usc and compact-valued if $\alpha=w$ (or $\alpha=m)$, and lsc if $\alpha=s$;

(iii) in $K_{1}(A, \Lambda) \times T\left(K_{1}(A, \Lambda), K_{2}\left(K_{1}(A, \Lambda), \Lambda\right), \Gamma\right) \times K_{2}\left(K_{1}(A, \Lambda), \Lambda\right) \times\left\{\mu_{0}\right\}$, the set $\{(x, t, y, \mu) \in A \times B \times A \times M \mid \rho(F(\bar{x}, t, y, \mu) ; C)\}$ is closed.

Then $\Psi_{\alpha \rho}$ is both usc and closed at $\left(\lambda_{0}, \gamma_{0}, \mu_{0}\right)$. 
Proof. Since $\alpha=\{w, m, s\}, \rho=\left\{\rho_{1}, \rho_{2}\right\}$, we have in fact six cases. However, the proof techniques are similar. We consider only the cases $\alpha=w, \rho=\rho_{1}$. Let relation $R$ be defined by $R(x, t, y, \mu)$ holds iff $F(\bar{x}, t, y, \mu) \subseteq C$. To apply Theorem 1 , we need to check only that in $K_{1}(A, \Lambda) \times T\left(K_{1}(A, \Lambda), K_{2}\left(K_{1}(A, \Lambda), \Lambda\right), \Gamma\right) \times K_{2}\left(K_{1}(A, \Lambda), \Lambda\right) \times\left\{\mu_{0}\right\}$, the set $\{(x, t, y, \mu) \in A \times B \times A \times M \mid F(\bar{x}, t, y, \mu) \subseteq C\}$ is closed.

Indeed, for all nets $\left\{\left(x_{n}, t_{n}, y_{n},{ }_{n}\right)\right\} \rightarrow\left(x_{0}, t_{0}, y_{0}, \mu_{0}\right)$ such that

$$
R\left(x_{n}, t_{n}, y_{n}, \mu_{n}\right) \text { holds. }
$$

By assumption (iii), we have

$$
F\left(x_{0}, t_{0}, y_{0}, \mu_{0}\right) \subseteq C
$$

Corollary 17 Assume for problem $\left(\mathrm{QEP}_{\alpha \rho}\right)$ that

(i) $E$ is lsc at $\lambda_{0}, K_{2}$ is usc and compact-valued in $K_{1}(A, \Lambda) \times\left\{\lambda_{0}\right\}$;

(ii) in $K_{1}(A, \Lambda) \times K_{2}\left(K_{1}(A, \Lambda), \Lambda\right) \times\left\{\gamma_{0}\right\}, T$ is usc and compact-valued if $\alpha=s$, and lsc if $\alpha=w$ (or $\alpha=m)$;

(iii) in $K_{1}(A, \Lambda) \times T\left(K_{1}(A, \Lambda), K_{2}\left(K_{1}(A, \Lambda), \Lambda\right), \Gamma\right) \times K_{2}\left(K_{1}(A, \Lambda), \Lambda\right) \times\left\{\mu_{0}\right\}$, the set $\{(x, t, y, \mu) \in A \times B \times A \times M \mid \bar{\rho}(F(x, t, y, \mu) ; C)\}$ is closed.

Then $\Psi_{\alpha \rho}$ is lower semicontinuous at $\left(\lambda_{0}, \gamma_{0}, \mu_{0}\right)$.

Proof. Since $\alpha=\{w, m, s\}, \rho=\left\{\rho_{1}, \rho_{2}\right\}$, we have in fact six cases. However, the proof techniques are similar. We consider only the cases $\alpha=s, \rho=\rho_{1}$. Let relation $R$ be defined by $R(x, t, y, \mu)$ holds iff $F(x, t, y, \mu) \subseteq C$. To apply Theorem 9 , we need to check only that in $K_{1}(A, \Lambda) \times T\left(K_{1}(A, \Lambda), K_{2}\left(K_{1}(A, \Lambda), \Lambda\right), \Gamma\right) \times K_{2}\left(K_{1}(A, \Lambda), \Lambda\right) \times$ $\left\{\mu_{0}\right\}$, the set $\left.\{(x, t, y, \mu) \in A \times B \times A \times M \mid F(x, t, y, \mu) \subseteq C)\right\}$ is closed.

Indeed, for all nets $\left\{\left(x_{n}, t_{n}, y_{n}, \mu_{n}\right)\right\} \rightarrow\left(x_{0}, t_{0}, y_{0}, \mu_{0}\right)$ such that

$$
R\left(x_{n}, t_{n}, y_{n}, \mu_{n}\right) \text { does not hold. }
$$

By assumption (iii), we have

$$
F\left(x_{0}, t_{0}, y_{0}, \mu_{0}\right) \not \subset C \text {. }
$$

$\square$

Corollary 18 Impose the assumption of Corollary 17 and the following additional conditions:

(iv) $K_{2}\left(., \lambda_{0}\right)$ is lsc in $K_{1}(A, \Lambda)$ and $E\left(\lambda_{0}\right)$ is compact;

(v) in $K_{1}(A, \Lambda) \times K_{2}\left(K_{1}(A, \Lambda), \Lambda\right), T\left(., ., \gamma_{0}\right)$ is usc and compact-valued if $\alpha=w$ (or $\alpha=m)$, and lsc if $\alpha=s$;

(vi) in $K_{1}(A, \Lambda) \times T\left(K_{1}(A, \Lambda), K_{2}\left(K_{1}(A, \Lambda), \Lambda\right), \Gamma\right) \times K_{2}\left(K_{1}(A, \Lambda), \Lambda\right)$, the set $\{(x, t, y) \in$ $\left.A \times B \times A \mid \rho\left(F\left(x, t, y, \mu_{0}\right) ; C\right)\right\}$ is closed.

Then $\Psi_{\alpha \rho}$ is Hausdorff lower semicontinuous at $\left(\lambda_{0}, \gamma_{0}, \mu_{0}\right)$.

Proof. Since $\alpha=\{w, m, s\}, \rho=\left\{\rho_{1}, \rho_{2}\right\}$, we have in fact six cases. However, the proof techniques are similar. We consider only the cases $\alpha=s, \rho=\rho_{1}$. Let relation $R$ be defined by $R(x, t, y, \mu)$ holds iff $F(x, t, y, \mu) \subseteq C$. To apply Theorem 12 , we need to check only that in $K_{1}(A, \Lambda) \times T\left(K_{1}(A, \Lambda), K_{2}\left(K_{1}(A, \Lambda), \Lambda\right), \Gamma\right) \times K_{2}\left(K_{1}(A, \Lambda), \Lambda\right)$, the set $\{(x, t, y) \in A \times$ $\left.\left.B \times A \mid F\left(x, t, y, \mu_{0}\right) \subseteq C\right)\right\}$ is closed. Indeed, for all nets $\left\{\left(x_{n}, t_{n}, y_{n}\right)\right\} \rightarrow\left(x_{0}, t_{0}, y_{0}\right)$ such that $R\left(x_{n}, t_{n}, y_{n}, \mu_{0}\right)$ holds. By assumption (vi), we have $F\left(x_{0}, t_{0}, y_{0}, \mu_{0}\right) \subseteq C$. $\square$ 
Remark 19 (i) Suppose that all conditions in Corollaries 16 and 17 are satisfied. Then, we have $\Psi_{\alpha}$ is both continuous and closed at $\left(\lambda_{0}, \gamma_{0}, \mu_{0}\right)$.

(ii) Suppose that all conditions in Corollaries 16 and 18 are satisfied. Then, we have $\Psi_{\alpha \rho}$ is Hausdorff continuous and closed at $\left(\lambda_{0}, \gamma_{0}, \mu_{0}\right)$.

\section{Acknowledgements}

The author is grateful to Prof. Phan Quoc Khanh and Dr. Lam Quoc Anh for their encour-agements in research. The author also thanks to the two anonymous referees for their valuable remarks and suggestions, which helped them to improve considerably the article.

\section{Competing interests}

The author declares that they have no competing interests.

Received: 1 January 2012 Accepted: 21 June 2012 Published: 21 June 2012

\section{References}

1. Anh, $L Q$, Khanh, PQ: Semicontinuity of solution sets to parametric quasivariational inclusions with applications to traffic networks I: upper semicontinuities. Set-Valued Anal. 16, 267-279 (2008). doi:10.1007/s11228-008-0074-z

2. Anh, LQ, Khanh, PQ: Semicontinuity of solution sets to parametric quasivariational inclusions with applications to traffic networks II: lower semicontinuities. Set-Valued Anal. 16, 943-960 (2008). doi:10.1007/s11228-008-0082-z

3. Khanh, PQ, Luc, DT: Stability of solutions in parametric variational relation problems. Set-Valued Anal. 16, 1015-1035 (2008). doi:10.1007/s11228-008-0101-0

4. Anh, LQ, Khanh, PQ: On the stability of the solution sets of general multivalued vector quasiequilibrium problems. J Optim Theory Appl. 135, 271-284 (2007). doi:10.1007/s10957-007-9250-9

5. Anh, LQ, Khanh, PQ: Semicontinuity of the solution sets of parametric multivalued vector quasiequilibrium problems. J Math Anal Appl. 294, 699-711 (2004). doi:10.1016/j.jmaa.2004.03.014

6. Anh, LQ, Khanh, PQ: Continuity of solution maps of parametric quasiequilibrium problems. J Glob Optim. 46, 247-259 (2010). doi:10.1007/s10898-009-9422-2

7. Bianchi, M, Pini, R: A note on stability for parametric equilibrium problems. Oper Res Lett. 31, 445-450 (2003). doi:10.1016/S0167-6377(03)00051-8

8. Bianchi, M, Pini, R: Sensitivity for parametric vector equilibria. Optimization. 55, 221-230 (2006). doi:10.1080/ 02331930600662732

9. Kimura, K, Yao, JC: Sensitivity analysis of solution mappings of parametric vector quasiequilibrium problems. J Glob Optim. 41, 187-202 (2008). doi:10.1007/s10898-007-9210-9

10. Kimura, K, Yao, JC: Sensitivity analysis of solution mappings of parametric generalized quasi vector equilibrium problems. Taiwan J Math. 9, 2233-2268 (2008)

11. Kimura, K, Yao, JC: Semicontinuity of solution mappings of parametric generalized vector equilibrium problems. J Optim Theory Appl. 138, 429-443 (2008). doi:10.1007/s10957-008-9386-2

12. Li, XB, Li, SJ: Continuity of approximate solution mappings for parametric equilibrium problems. J Glob Optim. 51, 541-548 (2011). doi:10.1007/s10898-010-9641-6

13. $\mathrm{Xu}, \mathrm{S}, \mathrm{Li}, \mathrm{SJ}$ : A new proof approach to lower semicontinuity for parametric vector equilibrium problems. Optim Lett. 3, 453-459 (2009). doi:10.1007/s11590-009-0124-y

14. Khanh, PQ, Luu, LM: Upper semicontinuity of the solution set of parametric multivalued vector quasivariational inequalities and applications. J Glob Optim. 32, 551-568 (2005). doi:10.1007/s10898-004-2693-8

15. Khanh, PQ, Luu, LM: Lower and upper semicontinuity of the solution sets and approximate solution sets to parametric multivalued quasivariational inequalities. J Optim Theory Appl. 133, 329-339 (2007). doi:10.1007/s10957-007-9190-4

16. Lalitha, CS, Bhatia, G: Stability of parametric quasivariational inequality of the Minty type. J Optim Theory Appl. 148 , 281-300 (2011). doi:10.1007/s10957-010-9755-5

17. Li, SJ, Chen, GY, Teo, KL: On the stability of generalized vector quasivariational inequality problems. J Optim Theory Appl. 113, 283-295 (2002). doi:10.1023/A:1014830925232

18. Muu, LD: Stability property of a class of variational inequalities. Math Operationsforsch Statist Ser Optim. 15, 347-351 (1984). doi:10.1080/02331938408842947

19. Wong, MM: Lower semicontinuity of the solution map to a parametric vector variational inequality. J Glob Optim. 46, 435-446 (2010). doi:10.1007/s10898-009-9447-6

20. Aubin, JP, Ekeland, I: Applied Nonlinear Analysis. John Wiley and Sons, New York (1984)

21. Berge, C: Topological Spaces. Oliver and Boyd, London (1963)

doi:10.1186/1687-1812-2012-102

Cite this article as: Van Hung: Continuity of solutions for parametric generalized quasi-variational relation

problems. Fixed Point Theory and Applications 2012 2012:102. 